\title{
Planejamento Regional Adaptativo em Sistemas Self-Adaptive de Larga Escala
}

\author{
Eudes S. Andrade, Gabriel F. Sousa, Sandro S. Andrade
}

Grupo de Pesquisa em Sistemas Distribuídos, Otimização, Redes e Tempo-Real (GSORT) Instituto Federal de Educação, Ciência e Tecnologia da Bahia (IFBA) Av. Araújo Pinho, no 39 - Canela - Salvador/BA - CEP: 40.110-150

\{eudesandrade, gabrielferreira, sandroandrade\}@ifba.edu.br

\begin{abstract}
Resumo. Com a crescente adoção de sistemas distribuídos de larga escala, tais como clusters para cloud computing, as atividades de implantação e configuração destas soluções se tornam consideravelmente mais complexas. Uma solução promissora é dotar tais sistemas com capacidades de autogerenciamento e diversos padrões de projeto para Sistemas Self-Adaptive estão disponíveis na literatura. Alguns são mais efetivos para operação em ambientes que sugerem a adoção de arquiteturas mais centralizadas. Outros apresentam melhores resultados ao operar de forma mais distribuída. Em ambientes altamente dinâmicos e incertos, no entanto, é difícil encontrar uma única solução efetiva para todos os cenários experimentados. Este trabalho apresenta um modelo adaptativo para implementação de Sistemas Self-Adaptive de larga escala que realizam autogerenciamento com diferentes graus de centralização. Os resultados obtidos mostram que o modelo apresenta desempenho satisfatório tanto em situações onde o sistema monitorado e o ambiente sugerem a adoção de topologias mais centralizadas, quanto em situações onde padrões mais descentralizados apresentam melhor custo-benefício.
\end{abstract}

\begin{abstract}
As large-scale distributed systems such as clusters for cloud computing become increasingly adopted, new challenges are posed when deploying and configuring such systems. A promising solution is to endow such systems with self-management capabilities and a number of design patterns for SelfAdaptive Systems is currently available. Some of those patterns are effective when operating in environments that require the adoption of centralized architectures, while others yield better results when controlling large-scale distributed systems. For highly dynamic and uncertain environments, though, a single solution is unlikely to provide effective control in every distinct scenario experienced by the system. This work presents an adaptive model for endowing large-scale Self-Adaptive Systems with self-management capabilities operating with a varying centralization degree. Results show that the proposed model presents improved performance in situations demanding both centralized and decentralized control topologies.
\end{abstract}

\section{Introdução}

Uma série de requisitos e características associados aos sistemas distribuídos modernos tem modificado a forma como tais aplicações são projetadas, desenvolvidas e avaliadas. 
Dentre tais características, destacam-se: a utilização de dispositivos multiprocessados, a disponibilidade de paralelismo em soluções altamente distribuídas, a necessidade de integração com outros sistemas e a capacidade de autogerenciamento em ambientes incertos [Northrop 2013]. A despeito da disponibilidade de diversas estratégias de Engenharia de Software para gerência da complexidade gerada por estes desafios, acredita-se que a capacidade humana de compreensão e manipulação de artefatos de software será um fator limitante em um futuro próximo [Huebscher and McCann 2008, Kephart and Chess 2003].

Tendo em vista que a maior parte dos desafios acima apresentados impactam diretamente os requisitos não-funcionais da aplicação - e que estes são amplamente influenciados por decisões arquiteturais tomadas em tempo de projeto - pesquisas recentes indicam que a transferência de decisões de projeto para o tempo de execução (runtime) é uma solução promissora no gerenciamento desta complexidade. Para tanto, os sistemas computacionais precisam apresentar alguma capacidade de autogerencimento [Huebscher and McCann 2008, Kephart and Chess 2003, Salehie and Tahvildari 2009]. Adicionalmente, características de Computação Ubíqua comumente presentes, por exemplo, em aplicações da Internet das Coisas e Cyber-Physical Systems, frequentemente demandam a necessidade de autogerenciamento. Um Sistema Self-Adaptive (SSA) é aquele que avalia o seu próprio comportamento e o modifica quando esta avaliação indica que: i) o seu propósito principal não está sendo efetivamente cumprido; ou ii) uma melhor funcionalidade e/ou desempenho pode ser alcançado [DARPA 1997].

Muitas arquiteturas para autogerenciamento estão atualmente disponíveis na literatura [Patikirikorala et al. 2012]. Ainda assim, a maioria delas apresenta desempenho satisfatório apenas em sistemas centralizados [Weyns 2010]. A dificuldade em alcançar autogerenciamento efetivo em ambientes descentralizados decorre do trade-off comumente encontrado em sistemas distribuídos: conhecimento do estado global do sistema vs. overhead de comunicação. Pode-se ilustrar esse trade-off analisando clusters para suporte a cloud computing. Em tais situações, quanto maior o overhead de comunicação (e, portanto, o conhecimento sobre o estado global do sistema), maior será a capacidade do sistema coordenar adaptações em caso de mudanças no ambiente. Por outro lado, um maior consumo de recursos pode diminuir a utilização útil do cluster para fins de computação. O objetivo da adoção de arquiteturas para autogerenciamento descentralizado, em tais cenários, é reagir ao trade-off que envolve a capacidade de atendimento da cloud frente às diversas configurações de ambiente apresentadas ao longo do tempo.

Este trabalho apresenta o projeto e avaliação de um modelo adaptativo para autogerenciamento em SSA de larga escala. Esse modelo prevê a reconfiguração dinâmica da topologia e forma de comunicação entre os múltiplos componentes locais de controle. Com esta abordagem, decisões de projeto acerca da topologia de controle a ser adotada são levadas para o tempo de execução. Com isso, obtém-se desempenho satisfatório de controle tanto em situações onde o sistema monitorado e o ambiente sugerem a adoção de topologias mais centralizadas, quanto em situações onde padrões completamente descentralizados apresentam um melhor trade-off. O mecanismo de reconfiguração dinâmica da arquitetura de controle aqui proposto foi modelado e avaliado via simulação de eventos discretos. Resultados indicam que houve uma economia estatisticamente significante de recursos computacionais ao utilizar o modelo proposto.

O restante deste trabalho está organizado como segue. A Seção 2 apresenta os 


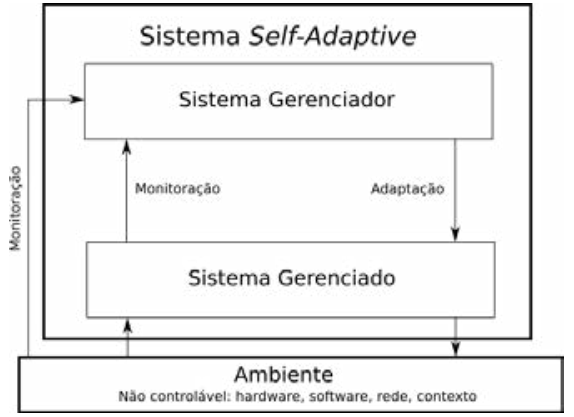

Figura 1. Elementos arquiteturais principais de um Sistema Self-Adaptive.

fundamentos sobre SSA e seus principais desafios. A Seção 3 explica o mecanismo para reconfiguração dinâmica de arquiteturas de controle aqui proposto. A Seção 4 apresenta detalhes sobre as avaliações via simulação realizadas, enquanto a Seção 5 discute e analisa os resultados obtidos. A Seção 6 apresenta os principais trabalhos relacionados e, finalmente, as conclusões e sugestões de trabalhos futuros são discutidos na Seção 7.

\section{Sistemas Self-Adaptive de Larga Escala}

O autogerenciamento é uma estratégia de projeto utilizada para os mais diversos fins. A despeito disto, a maior parte destas soluções realiza a migração de decisões - anteriormente tomadas em tempo de projeto - para o tempo de execução. Com este artifício, busca-se minimizar possíveis inflexibilidades/ineficiências decorrentes de comprometimentos prévios com determinadas decisões arquiteturais. A ocorrência de situações não previstas pelo arquiteto de software é consequência, sobretudo, do dinamismo dos ambientes de execução das aplicações.

Uma definição de SSA comumente adotada na literatura é aquela proposta em [DARPA 1997]: um SSA é aquele que avalia o seu próprio comportamento e o modifica quando esta avaliação indica que: $i$ ) o seu propósito principal não está sendo efetivamente cumprido; ou ii) uma melhor funcionalidade e/ou desempenho podem ser alcançados [DARPA 1997]. Um SSA é geralmente caracterizado pela presença de dois elementos arquiteturais principais, ilustrados na Figura 1. O primeiro - denominado sistema gerenciado - é o elemento responsável pela implementação das regras de negócio da aplicação. O segundo elemento - denominado sistema gerenciador - é responsável pela implementação das ações necessárias para a realização das adaptações.

Embora diferentes mecanismos para autogerenciamento tenham sido propostos nos últimos anos, a maior parte deles baseia-se na utilização de algum tipo de loop de adaptação. Uma arquitetura de referência amplamente adotada no projeto destes loops de adaptação é a arquitetura MAPE-K [Kephart and Chess 2003]. Esta arquitetura define a execução, em sequência, das seguintes fases: Monitoramento, Análise, Planejamento e Execução - com o auxílio de uma base de conhecimento (Knowledge Base). A fase de monitoramento é responsável pela obtenção de informações sobre o estado atual do sistema/ambiente. A atividade de análise realiza a avaliação das informações obtidas na fase de monitoramento. Após a avaliação destes dados, delibera-se pela necessidade ou não da realização de uma adaptação. A fase de planejamento é responsável pela definição de quais atividades de adaptação devem ser realizadas, com o objetivo que o sistema retorne a 


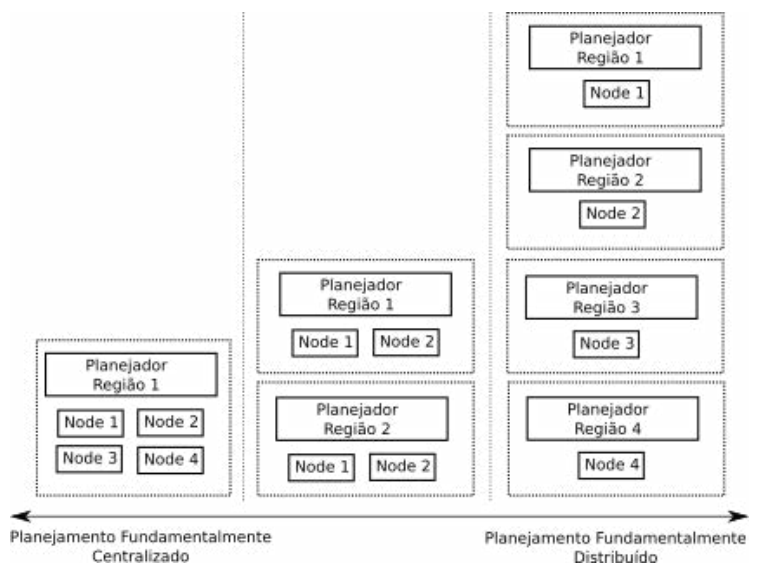

Figura 2. Diferentes instâncias do padrão Regional Planning.

um estado particular de interesse. Por fim, a atividade de execução realiza a implantação, no sistema gerenciado, das ações de adaptação definidas na fase de planejamento.

Em ambientes altamente distribuídos, heterogêneos e complexos, um único loop de controle pode não ser suficiente para gerenciar toda a sua estrutura [Weyns 2010]. Nestes cenários, portanto, múltiplos loops devem ser empregados para controlar as diferentes partes (múltiplos sistemas gerenciados) que compõem o sistema. Esta associação de loops de controle aos diversos nós do sistema distribuído traz uma complexidade a mais durante o projeto da solução: a coordenação e interação entre estes múltiplos loops.

Em [Weyns 2010], um conjunto de seis padrões arquiteturais para implementação de autogerenciamento descentralizado é apresentado. Para cada padrão, são apresentados os atributos de qualidade decorrentes da sua adoção e as restrições impostas pelas decisões que compõem a solução. Um dos padrões apresentados é o Regional Planning - recomendado para situações onde diversas partes integradas (regiões) de um sistema demandam não apenas adaptações locais (dentro das regiões) mas também adaptações globais (que extrapolam os limites das regiões). A solução proposta pelo Regional Planning prevê um único componente $\mathrm{P}$ (planning) para cada região. Este planejador regional é responsável por coletar as informações de todos os componentes que fazem parte da sua região, para que o planejamento seja realizado. Planejadores regionais coordenam entre si (sobre múltiplas regiões) para a realização do planejamento da adaptação global.

A Figura 2 apresenta diferentes instâncias do padrão Regional Planning, com diferentes tamanhos de região. Na figura, dois tipos de nós de controle são apresentados. $O$ primeiro deles apresenta apenas o componente $P$ (planejador da região) e cada região é controlada por apenas um nó do tipo P. O segundo tipo apresenta os outros três componentes MAPE-K restantes: MAE (Monitoramento, Análise e Execução). Em cada região, existem múltiplos nós de controle do tipo $\mathrm{MAE}$, que realizam o monitoramento do seu sistema local e do seu ambiente de execução. O componente A realiza uma análise local dos dados monitorados e reporta resultados para o planejador $\mathrm{P}$ da sua região. O planejador regional $P$, por sua vez, planeja a adaptação local da região após cooperar com os planejadores $P$ das outras regiões. Com este arranjo, a adaptação realiza a busca por adaptações globais, extrapolando os limites do monitoramento e análise locais.

Como consequência da adoção do padrão Regional Planning, espera-se uma 
redução na quantidade de dados trafegados, uma vez que o monitoramento e análise são realizadas localmente. A análise local dos dados monitorados reduz a quantidade de dados e a frequência com que interações serão realizadas com o nó central da região. Por outro lado, a necessidade de agregação local - no nó de planejamento - dos resultados das diversas operações de análise pode implicar em overhead excessivo. Outra desvantagem é a necessidade de uma fase de planejamento demasiadamente detalhada, uma vez que as fases de execução não coordenam. Finalmente, a adoção do padrão Regional Planning requer a definição - em tempo de projeto - do tamanho de região com o qual o sistema será configurado. Consequentemente, ambientes diferentes podem demandar diferentes tamanhos de região para que o autogerenciamento apresente desempenho satisfatório. Em ambientes altamente dinâmicos e incertos, autogerenciamento satisfatório só é obtido com a variação - em tempo de execução - do tamanho de região adotado.

\section{O Mecanismo de Planejamento Regional Adaptativo}

Este trabalho apresenta o projeto, implementação e avaliação de um modelo adaptativo para autogerenciamento em SSA de larga escala. O modelo permite a reconfiguração dinâmica da topologia do sistema gerenciador e da forma de comunicação entre os múltiplos componentes locais do controle. Com esta abordagem, decisões referentes à topologia de controle a ser adotada (tamanho da região de planejamento) são levadas para o tempo de execução. Com isso, espera-se obter desempenho satisfatório de autogerenciamento tanto em situações onde o sistema gerenciado/ambiente sugerem a adoção de topologias mais centralizadas, quanto em situações onde padrões completamente descentralizados são mais adequados. Em ambientes altamente dinâmicos e incertos, é comum que uma determinada topologia - adequada para um dado cenário - não seja mais satisfatória após a ocorrência de mudanças no ambiente.

Este trabalho viabiliza o projeto de sistemas gerenciadores de tal modo que eles mesmos sejam tratados como SSA. Dessa forma, decisões acerca dos limites das regiões em arquiteturas que seguem o padrão Regional Planning não mais são realizadas off-line. Com o modelo proposto, em função do seu estado interno e das condições do ambiente, a arquitetura do sistema gerenciador será reconfigurada para adotar desde abordagens mais centralizadas até os extremos providos pelos padrões mais distribuídos.

\subsection{Motivação}

Conforme discutido na Seção 2, a adoção do padrão Regional Planning apresenta um trade-off que envolve a qualidade da adaptação vs. a quantidade de dados trafegados (e localmente mantidos) nos nós responsáveis pelo planejamento. Este trade-off é comumente administrado pelo arquiteto de software, em tempo de projeto. Para tanto, os tamanhos das regiões são fixos e definidos antecipadamente, sobretudo considerando as situações de maior sobrecarga previstas para a operação do sistema.

Visto que no padrão Regional Planning o tamanho das regiões é definido em tempo de projeto, os sistemas gerenciadores tendem a operar de forma não-ótima quando as condições ambientais se desviam daquelas consideradas em tempo de projeto. Para situações de sobrecarga do sistema - onde um máximo de controle se faz necessário os controles regionais atuam de maneira satisfatória. Por outro lado, em situações onde a utilização de tamanhos menores de região seria suficiente para garantir a qualidade de operação do sistema, um superprovisionamento de recursos estará sempre presente. 
A possibilidade de utilização de um modelo onde a decisão acerca do tamanho da região não mais seja uma definição a ser administrada em tempo de projeto - mas sim uma decisão levada para o tempo de execução - é o que motiva a adoção do Planejamento Regional Adaptativo proposto neste trabalho. Ao utilizar um tamanho variável de região, o sistema gerenciador terá a possibilidade de operar de maneira ótima, independente dos diferentes estados apresentados pelo ambiente e sistema gerenciado ao longo do tempo.

\subsection{O Mecanismo Proposto}

O Planejamento Regional Adaptativo aqui proposto implementa uma variação do padrão Regional Planning. Diferente do Regional Planning convencional, o Planejamento Regional Adaptativo transfere para o tempo de execução a decisão acerca do tamanho das regiões. Dessa forma, o sistema gerenciador passa a ser, ele mesmo, um SSA que rejeita perturbações no ambiente através da variação no tamanho das regiões de planejamento.

Para isso, um segundo nível de controle (metacontrole) é inserido na arquitetura. Os tamanhos de região que antes eram fixos - definidos off-line de maneira a suportar os cenários de pior caso - passam a ser agora mantidos em tempo de execução pelo metacontrole. Com isso, espera-se obter desempenho satisfatório de autogerenciamento tanto em situações onde o sistema gerenciado/ambiente sugerem a adoção de regiões maiores (planejamento global), quanto em situações onde o uso de pequenas regiões (planejamento local) já é suficiente. Trata-se, portanto, de adicionar ao padrão Regional Planning um segundo nível de adaptação.

Em momentos onde o sistema gerenciado e o ambiente demandem menos da adaptação, o tamanho das regiões será diminuído pelo metacontrole. Dessa forma, recursos serão poupados. Por outro lado, em momentos onde o metacontrole verifica que o componente MAPE-K responsável pelo planejamento regional necessita de monitoramento e análises mais globais - de forma a garantir os requisitos de qualidade acordados - o tamanho das regiões é aumentado. A fundamentação para tal investigação envolve os impactos decorrentes da variação do tamanho das regiões. A hipótese aqui investigada considera que a adoção de regiões grandes traz como vantagem a maior presença de informações globais, o que resulta na possibilidade de realização de adaptações mais efetivas. Por outro lado, a adoção de regiões pequenas beneficia aspectos tais como disponibilidade e escalabilidade. Sendo assim, o objetivo geral da solução aqui apresentada é regular, de forma automática, o trade-off existente entre a efetividade das adaptações vs. a disponibilidade e escalabilidade da solução.

Algumas premissas foram consideradas nesta proposta. Primeiro, assume-se que o overhead introduzido pelo metacontrole é desprezível. Visto que as operações de reconfiguração geralmente envolvem a modificação de parâmetros, tais como tamanho de buffers ou thread pools, o custo do trânsito de tais parâmetros na rede é consideravelmente menor que transitar os dados da aplicação. Segundo, todas as regiões possuem sempre o mesmo tamanho. Embora tal premissa eventualmente não seja verificada em clusters com bastante heterogeneidade, suportar diferentes tamanhos de região traria uma complexidade maior ao modelo e será analisado em trabalhos futuros. Por fim, considera-se que o cluster possui jobs sendo demandados a todo momento, o que viabiliza o contínuo monitoramento do desempenho das aplicação e do autogerenciamento. 


\section{Avaliação}

O mecanismo de Planejamento Regional Adaptativo aqui proposto foi modelado e avaliado via simulação de eventos discretos. Os resultados da simulação foram avaliados considerando dois grupos de controle: o primeiro, decorrente da execução da simulação utilizando o Planejamento Regional Adaptativo proposto neste trabalho; e o segundo, utilizando o padrão Regional Planning convencional, com o uso de um tamanho fixo de região de planejamento. As seguintes hipóteses foram investigadas no experimento:

- Hipótese alternativa $\left(H_{1}\right)$ : mantida a mesma qualidade do serviço, o tamanho médio da região (e, portanto, o consumo de recursos) ao utilizar o Planejamento Regional Adaptativo aqui proposto $\left(T_{P R A}\right)$ é menor que o tamanho médio da região ao utilizar o Regional Planning convencional $\left(T_{P R}\right)$.

- Hipótese nula $\left(H_{0}\right)$ : mantida a mesma qualidade do serviço, o tamanho médio da região (e, portanto consumo de recursos) ao utilizar o Planejamento Regional Adaptativo aqui proposto $\left(T_{P R A}\right)$ não é menor que o tamanho médio da região ao utilizar o Regional Planning convencional $\left(T_{P R}\right)$.

\subsection{Simulação}

O modelo da simulação define o sistema gerenciado, gerenciador e metacontrole como um único sistema distribuído. Este sistema distribuído é modelado como um grafo $E(N, C)$. O grafo $E$ é composto por um conjunto finito de nós, denominado $N=\left\{N_{1}, N_{2}, \ldots, N_{n}\right\}$ e um conjunto finito de canais de comunicação $C=f\left(N_{i}, N_{j}\right) \mid N_{i}, N_{j} \in N$. Vértices representam nós (sistemas gerenciados e gerenciadores) e as arestas representam canais de comunicação. $O$ grafo $E$ implementa os detalhes referentes ao sistema simulado, tais como os componentes que simulam os nós do sistema, informações acerca das regiões e eventuais perturbações $(P)$ presentes no ambiente.

Uma vez que os nós $N$ têm o seu tempo de serviço afetado diretamente pela qualidade da adaptação, tem-se que o tempo de serviço das operações (TS) é função do tamanho das regiões $(R)$. Além do tamanho das regiões, nota-se que o tempo de serviço das operações é diretamente influenciado pela perturbação $(P)$ presente no ambiente. Dessa forma, tem-se que $T S$ é função tanto do tamanho das regiões quanto da perturbação existente no ambiente: $T S(R, P)$. A perturbação $(P)$ foi modelada através de uma variável aleatória, cuja intensidade é obtida utilizando a distribuição normal, ao passo que sua frequência de ocorrência é denotada por uma distribuição exponencial.

Além das relações definidas na função $T S(R, P)$, uma variável aleatória $K$ foi inserida no cálculo do tempo de serviço das operações para representar componentes estocásticos internos ao nó. Os valores de $K$ são obtidos de acordo com a distribuição Erlang [Jain 1991]. E armazena ainda a fila de operações a ser executada. O modelo proposto considera a existência de uma fila de operações constantemente alimentada e representa jobs demandados por usuários. Os nós presentes no sistema atuam consumindo as operações que aguardam por processamento. O tempo de chegada de novas operações na fila foi modelado através de uma variável aleatória com distribuição exponencial.

Dois níveis de adaptação são observáveis no modelo proposto. O primeiro nível implementa o padrão Regional Planning e é responsável por garantir a adaptação nos nós. Esse controle é implementado na função que calcula o tempo de serviço das operações 
$T S(R, P)$. O tamanho das regiões $(R)$ é a variável que representa a contribuição, do controle de primeiro nível, no tempo de serviço das operações. O segundo nível implementa a operação de metacontrole $(M C)$, responsável por adaptar os controladores de primeiro nível, sobretudo atuando no tamanho das suas regiões. O $M C$ atua no tamanho das regiões da seguinte forma: se a quantidade de ciclos idle realizados é maior que um determinado valor de referência, o tamanho das regiões é reduzido. Por outro lado, caso a quantidade de ciclos idle mantenha-se abaixo do valor de referência, o $M C$ atuará aumentando o tamanho das regiões. Apesar de o modelo não depender diretamente da lei de controle, para que a simulação pudesse ser executada fez-se necessário optar por uma implementação concreta. Nesta simulação, optou-se por utilizar controladores PID (Proporcional-Integral-Derivativo) [Hellerstein et al. 2004] para realização da adaptação.

A simulação foi implementada utilizando a linguagem de programação Python e o framework para simulação SimPy (http://simpy.readthedocs.org). Pacotes adicionais foram utilizados para geração dos números aleatórios de acordo com as distribuições definidas no modelo. A análise dos resultados foi realizada com a ferramenta $\mathrm{R}$ (http://www.rproject.org), com o auxílio de módulos para execução dos testes estatísticos.

\subsection{Análise dos Resultados}

A análise dos dados foi realizada com base em um estudo comparativo com dois diferentes grupos de dados. O primeiro grupo representou a execução da simulação utilizando o padrão Regional Planning convencional. O segundo grupo, por sua vez, representou a execução da simulação com a utilização do Planejamento Regional Adaptativo proposto neste trabalho. O experimento apresentou um único fator: o tamanho das regiões em tempo de execução. Esse único fator apresenta dois diferentes níveis: Planejamento Regional Adaptativo (PRA) ou Regional Planning convencional (PR).

Para cada nível foram executadas cinquenta replicações utilizando as mesmas sementes de geração de valores para as variáveis aleatórias. A quantidade de ciclos idle foi medida para cada uma das replicações. Os resultados das cinquenta replicações foram inicialmente analisados com os testes de Anderson-Darling e Levene, com o objetivo de analisar a normalidade e homoscedasticidade dos resultados. Com base nos resultados destes testes, decidiu-se pela avaliação da hipótese nula usando ou testes paramétricos ( $t$-test) ou testes não-paramétricos (Wilcoxon).

\section{Resultados}

Para realização dos testes considerando o nível $P R$ (tamanho fixo das regiões) foi necessário definir um tamanho fixo para as regiões. Este tamanho foi o mínimo necessário para que o controle tivesse condições de adaptar o sistema satisfatoriamente na situação de pior caso. Uma vez que o tempo de serviço $T S$ é função do tamanho da região e do nível de perturbação verificado em dado instante, calculou-se o tamanho mínimo de região necessário para suportar o ambiente nos momentos de maior perturbação possível. A perturbação gerada no ambiente é uma variável aleatória com distribuição normal. Logo, considerou-se como perturbação máxima o seu valor médio somado ao erro máximo (valor de referência - valor observado). A segunda execução da simulação foi realizada considerando o nível $P R A$ (tamanho variável das regiões). A configuração inicial da simulação inicia o tamanho da região com o mesmo valor utilizado no nível $P R$, porém, com o decorrer da simulação, o tamanho da região é administrado pelo metacontrole. 

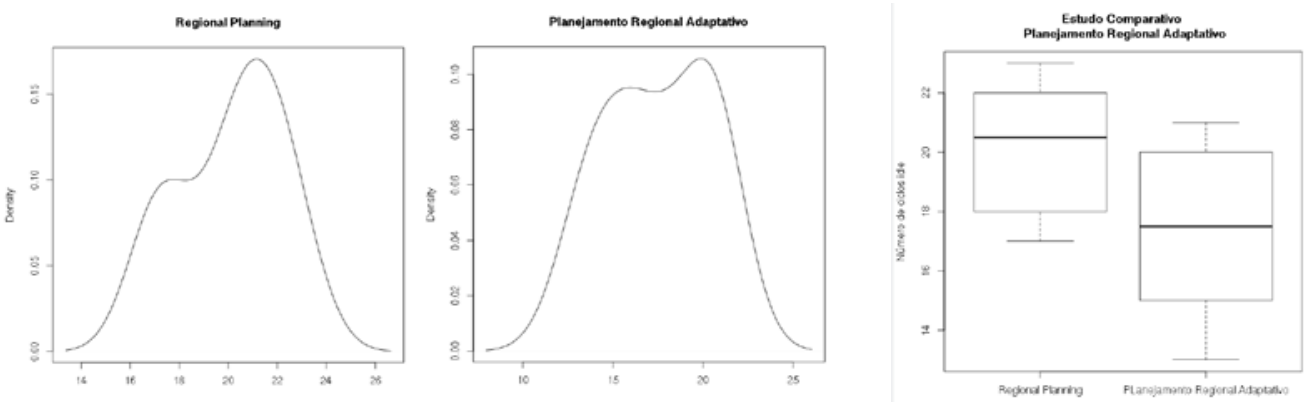

Figura 3. Distribuição dos dados resultantes da simulação.

Uma investigação acerca da normalidade e homoscedasticidade dos dados foi realizada, com o objetivo de decidir se a hipótese nula do experimento poderia ser analisada através de um teste paramétrico ( $t$-test). O resultado da análise de normalidade, com um nível de significância $\alpha=0.01$, apresentou um $p$-value igual a 0.85 . A análise de homoscedasticidade apresentou um $p$-value igual a 0.05. Dos resultados anteriores, conclui-se que ambos os testes falharam e, portanto, testes não-paramétricos devem ser utilizados. A Figura 3 apresenta os resultados obtidos.

Considerando as características de normalidade e homoscedasticidade dos dados, concluiu-se que o t-test não poderia ser utilizado como ferramenta para investigação da hipótese nula. Optou-se então pela utilização do teste de Wilcoxon (não-paramétrico). O resultado do teste de Wilcoxon para as amostras anteriores apresentou, com um nível de significância $\alpha=0.01$, um $p$-value igual a 0.85 . O resultado obtido indica que a hipótese nula $H_{0}$ pode ser rejeitada e, portanto, que as amostras avaliadas apresentam melhora significativa dos resultados. Uma representação gráfica do estudo comparativo dos resultados é apresentada na Figura 3.

\section{Trabalhos Relacionados}

Diferentes padrões de projeto podem ser encontrados na literatura para tentar resolver o problema do planejamento [Abuseta and Swesi 2015, Weyns 2010]. Um destes padrões de projeto é o "Information Sharing", que restringe a comunicação entre os componentes presentes no padrão descentralizado. Nesta organização, apenas o componente de monitoramento M pode se comunicar com outros componentes externos. Esta abordagem resulta em soluções com melhor escalabilidade de comunicação do que o controle totalmente descentralizado, agilizando o processo de execução da adaptação. Porém, este padrão não é indicado para ambientes altamente dinâmicos pois, como as análises e planejamentos são feitos localmente, as adaptações acabam sendo sub-ótimas.

Outra possibilidade é o padrão de projeto denominado controle hierárquico. Esta abordagem define uma separação de concerns por camadas, onde cada nível executa seu próprio loop MAPE-K. Os níveis inferiores, com seus componentes de monitoramento e execução, lidam diretamente com o subsistema gerenciado. A camada mais alta se preocupa com a adaptação geral do sistema. $\mathrm{O}$ padrão de controle hierárquico permite gerenciar a complexidade do SSA. Porém, esta abordagem não é indicada para ambientes em constante mudança pois a divisão em camadas permite que existam dois planejamentos de adaptação, sendo eles global e específico. 


\section{Conclusão}

Este trabalho apresentou o Planejamento Regional Adaptativo - uma extensão do padrão arquitetural Regional Planning que promove uma economia de recursos computacionais e menor necessidade de provisionamento de ambiente em SSA de larga escala. Diferente do padrão Regional Planning, o modelo proposto dotou, tanto o sistema gerenciador quanto o sistema gerenciado, de capacidades de autogerenciamento. Com isso, viabiliza-se a execução do sistema gerenciador utilizando tamanhos reduzidos de regiões, o que implica em uma economia de recursos. O mecanismo foi modelado e avaliado via simulação de eventos discretos. Dois grupos foram comparados: controle utilizando o padrão Regional Planning convencional e controle utilizando o Planejamento Regional Adaptativo proposto. A análise dos resultados indica que, com um nível de significância $\alpha=0.01$, houve um aumento de desempenho do sistema gerenciador ao utilizar o Planejamento Regional Adaptativo.

\section{Referências}

[Abuseta and Swesi 2015] Abuseta, Y. and Swesi, K. (2015). Design patterns for self adaptive systems engineering. CoRR, abs/1508.01330.

[DARPA 1997] DARPA, B. A. A. (1997). Self-adaptive software. Technical Report 98-12, DARPA (Defense Advanced Research Projects Agency).

[Hellerstein et al. 2004] Hellerstein, J. L., Diao, Y., Parekh, S., and Tilbury, D. M. (2004). Feedback Control of Computing Systems. John Wiley \& Sons.

[Huebscher and McCann 2008] Huebscher, M. C. and McCann, J. A. (2008). A survey of autonomic computing - degrees, models, and applications. ACM Comput. Surv., 40(3).

[Jain 1991] Jain, R. (1991). The art of computer systems performance analysis: techniques for experimental design, measurement, simulation, and modeling. SIGMETRICS Performance Evaluation Review, 19:5-11.

[Kephart and Chess 2003] Kephart, J. O. and Chess, D. M. (2003). The vision of autonomic computing. IEEE Computer, 36(1):41-50.

[Northrop 2013] Northrop, L. M. (2013). Does scale really matter? ultra-large-scale systems seven years after the study (keynote). In Notkin, D., Cheng, B. H. C., and Pohl, K., editors, 35th International Conference on Software Engineering, ICSE '13, San Francisco, CA, USA, May 18-26, 2013, page 857. IEEE / ACM.

[Patikirikorala et al. 2012] Patikirikorala, T., Colman, A., Han, J., and Wang, L. (2012). A systematic survey on the design of self-adaptive software systems using control engineering approaches. In Software Engineering for Adaptive and Self-Managing Systems (SEAMS), pages $33-42$.

[Salehie and Tahvildari 2009] Salehie, M. and Tahvildari, L. (2009). Self-adaptive software: Landscape and research challenges. TAAS, 4(2).

[Weyns 2010] Weyns, D. (2010). On patterns for decentralized control in self-adaptive systems. In de Lemos, R., Giese, H., Müller, H. A., and Shaw, M., editors, Software Engineering for Self-Adaptive Systems II, volume 7475 of Lecture Notes in Computer Science, pages 76-107. Springer. 